\title{
An Ontology for Designing Models of Epidemics
}

\author{
Geoff Frank, Bill Wheaton, Vesse Bakalov, \\ Phil Cooley, Diane Wagener
}

24 July 2009

This work was supported by the National Institute of General Medical Sciences MIDAS Program (Grant \#5U01GM70698-5)

3040 Cornwallis Road D P.O. Box 12194 - Research Triangle Park, North Carolina, USA 27709 Phone 919-541-6629 Fax 919-541-8830 e-mail gaf@rti.org 


\section{Role of the Ontology in Building Models}

- Help researchers find relevant models, simulations, and results

- Help modelers find relevant data to build, calibrate, and validate their models

- Use taxonomies that are accepted by the community as a means of accessing data at multiple levels of abstraction 


\section{MIDAS}

\section{HARVARD MEDICINE}

HARVARD SCHOOL OF PUBLIC HEALTH

Paig Penn

Veterinary Medicine

University of Pittsburgh

\section{UCIrvine}

\section{感VirginiaTech}

- The Models of Infectious Disease Agent Study (MIDAS) formed in May of 2004 by NIGMS

- The National Institute of General Medical Sciences is one of the NIH institutes

- The NIGMS supports basic biomedical research that increases understanding of life processes and lays the foundation for advances in disease diagnosis, treatment, and prevention.

- NIGMS contact for MIDAS: Dr. Irene Eckstrand 


\section{MIDAS Results}

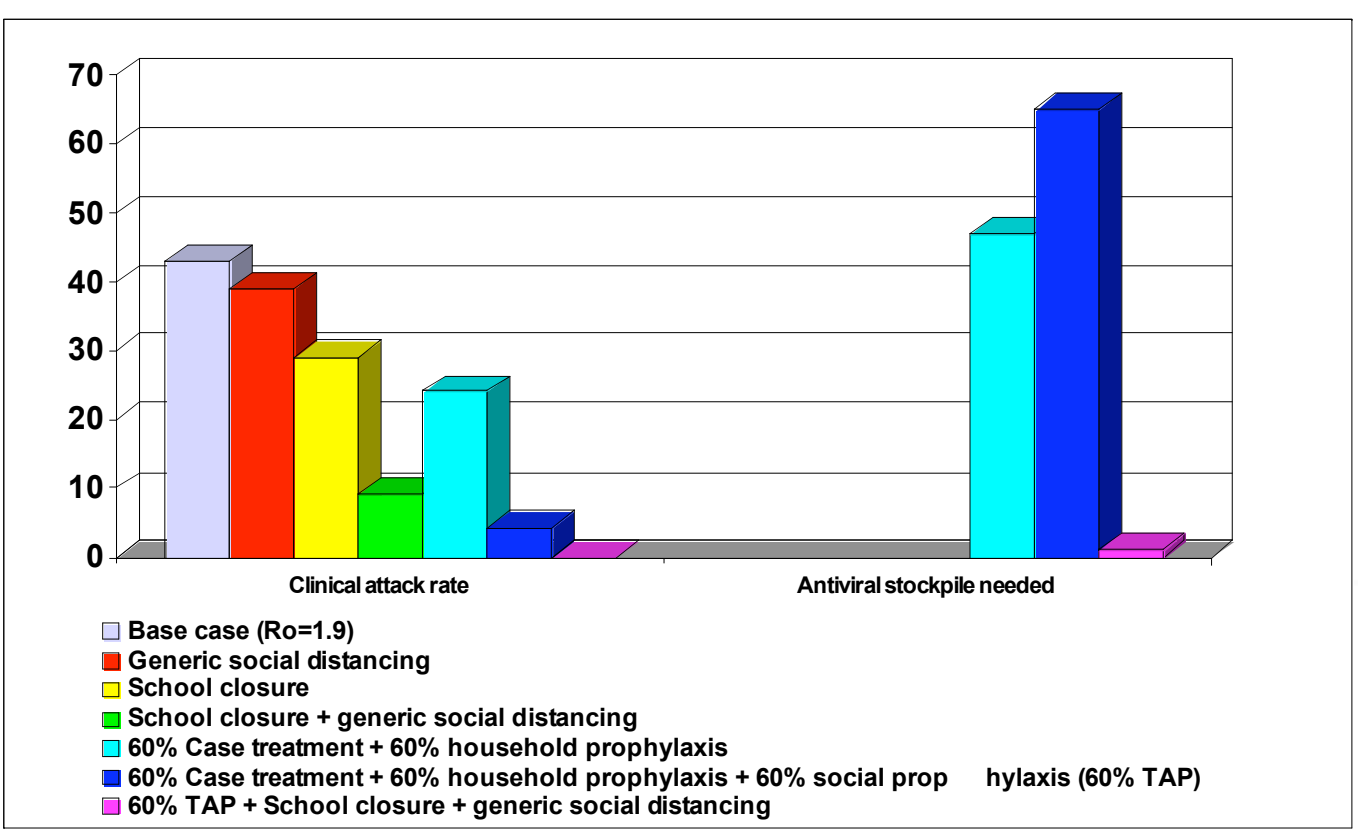

From Longini et al., 2005
- Analysis of air travel restrictions to prevent pandemics

- Analysis of targeted intervention strategies for controlling avian flu pandemics for the US Department of Homeland Security

- Analysis of vaccine and antiviral medicine stockpiling for the World Health Organization 


\section{Components of an Epidemic Model}

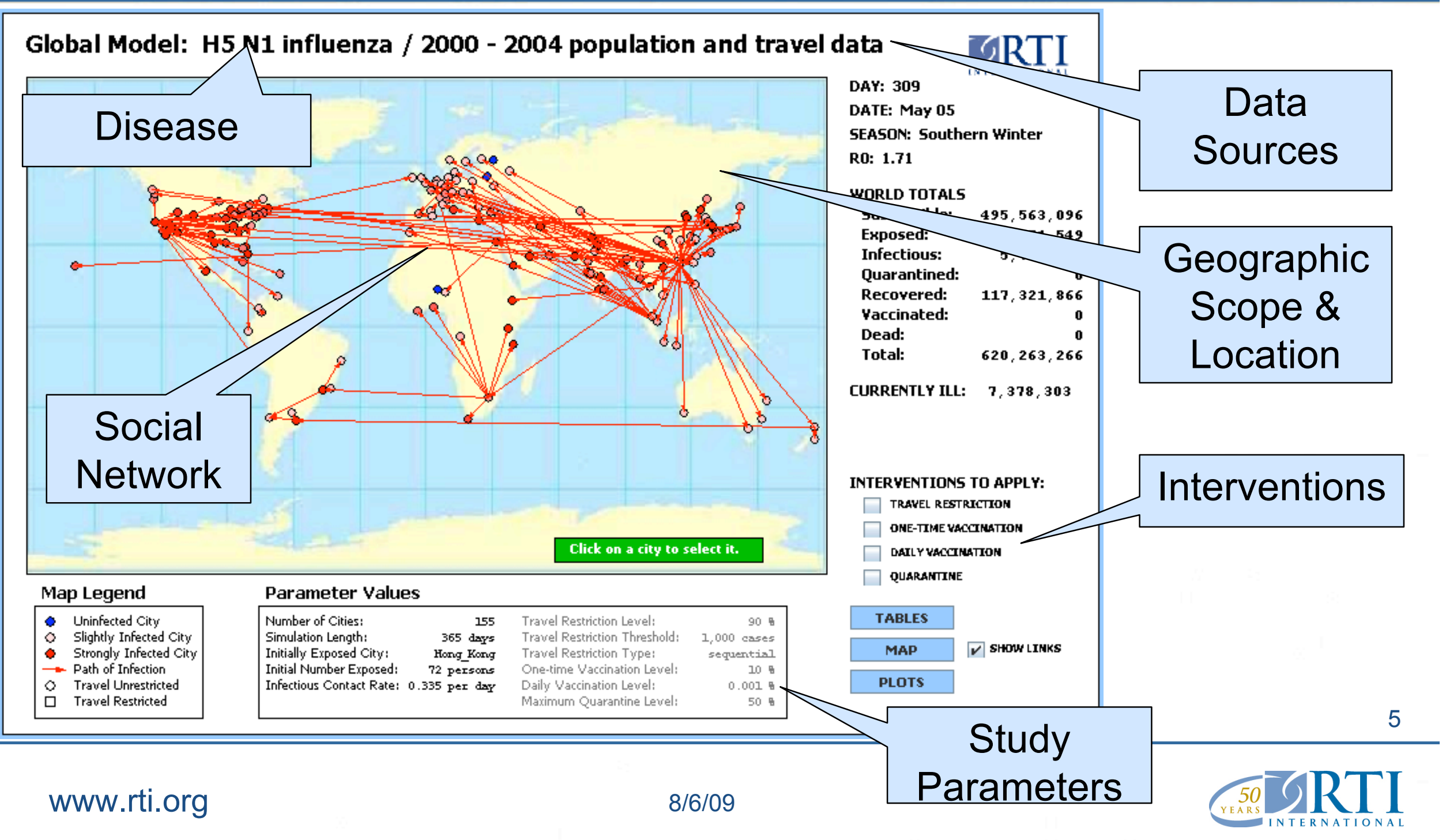




\section{The Disease Model}

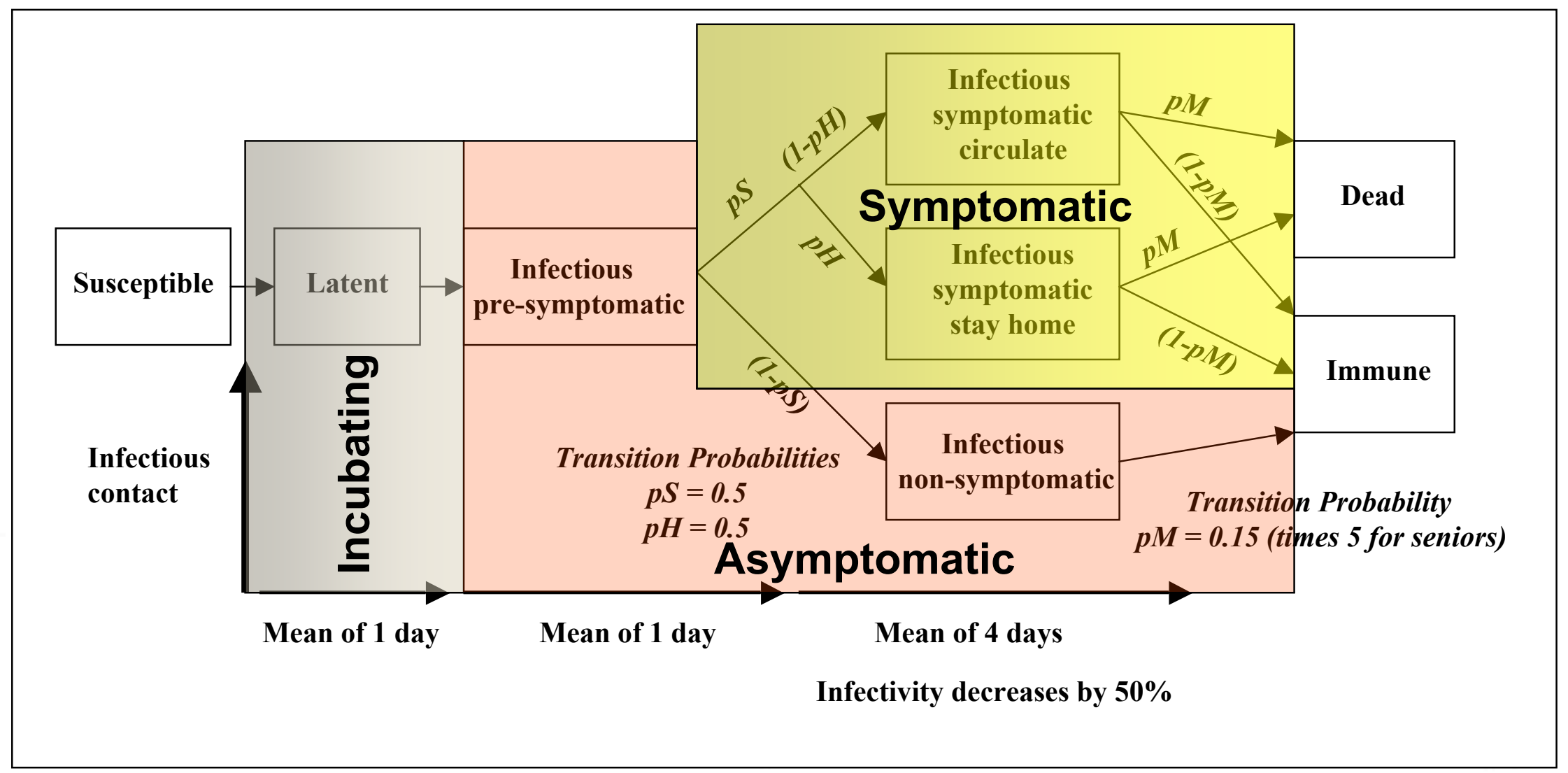

A Compromise between Ferguson 2005, Nature; Longini et al., 2005, Science; and Longini et al., 2004, Am J Epidemiology 


\section{A Geo-Referenced Social Network Model}

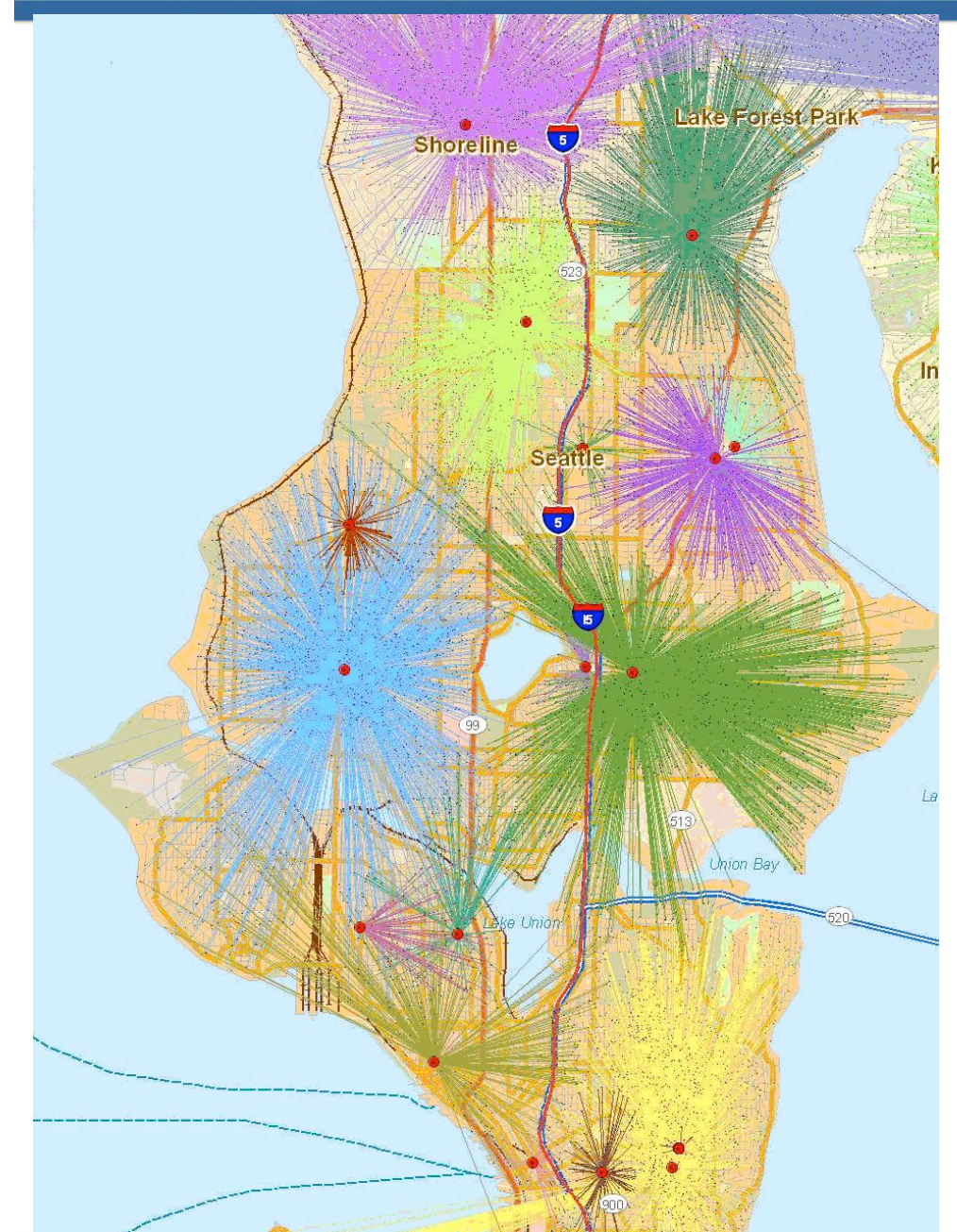

- Households

- Household population

- Workplaces

- Workplace mapping

- Hospitals, schools, \& hotels

- Schools

- School assignments

- Communities

- Hospitals

- Hotels

- Group Quarters

- Dormitories, barracks, prisons, nursing homes 


\section{Intervention Models}

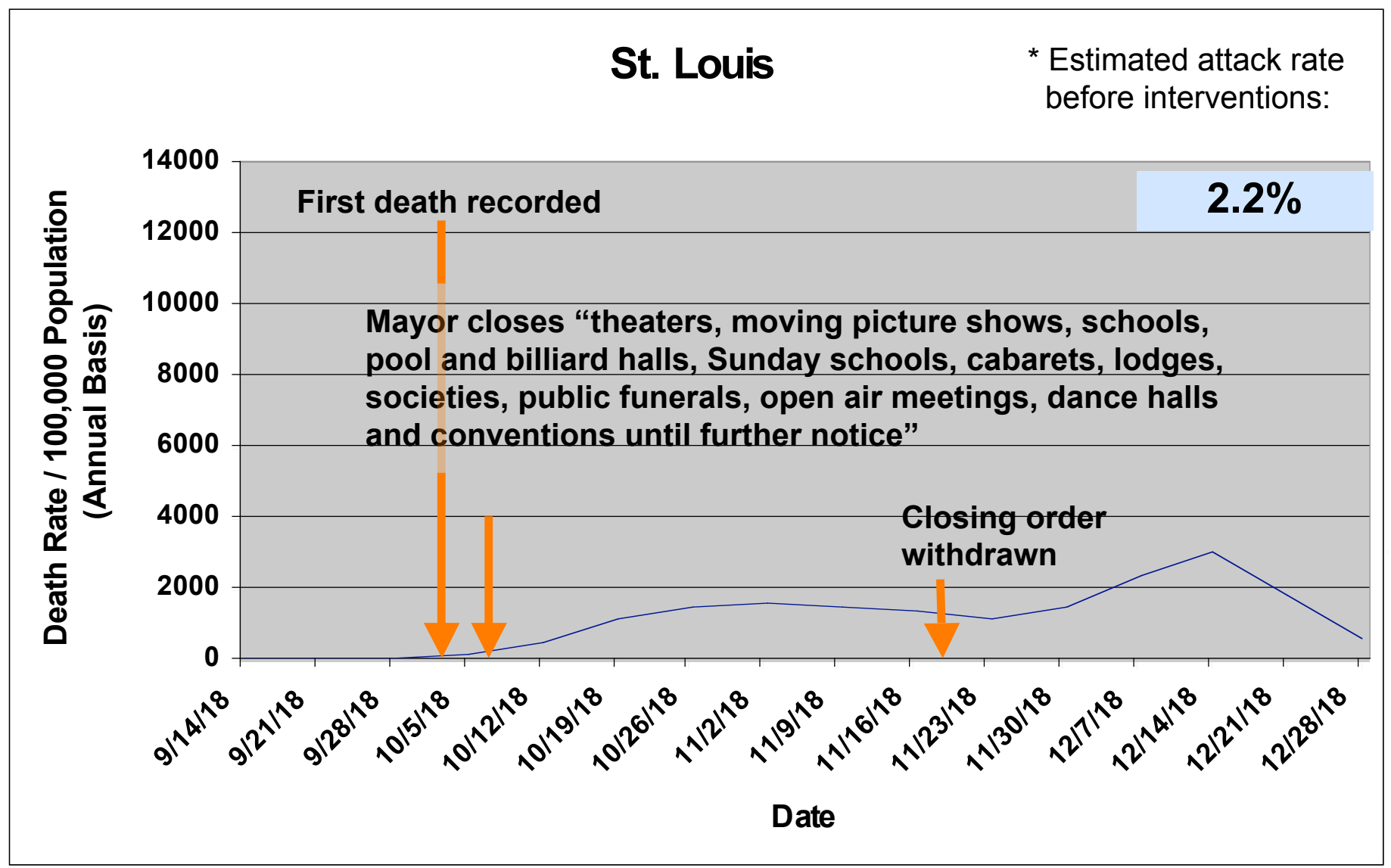




\section{Study Design: Parameters and Output}

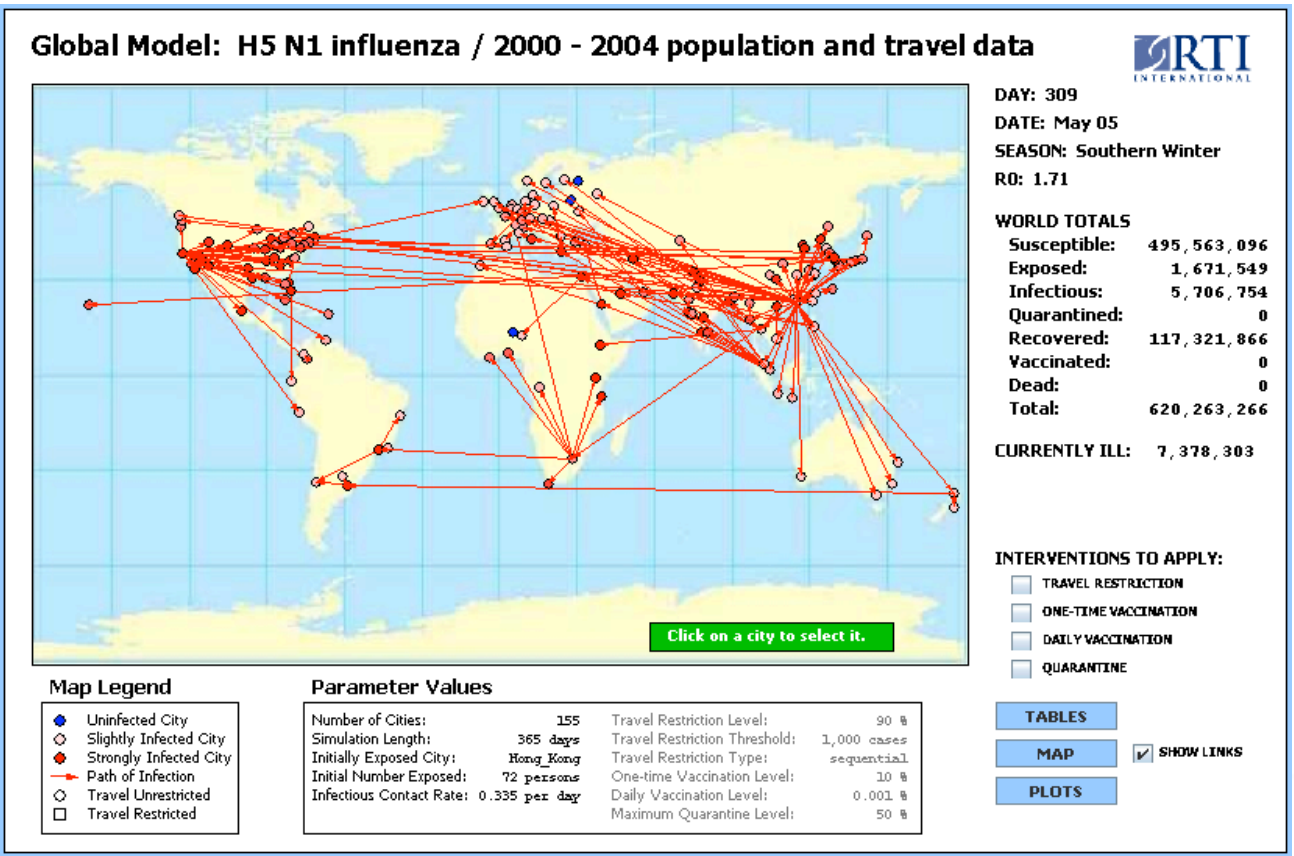

- Initial Conditions

- Initial infection location

- Infection rates $\left(R_{0}\right)$

- Time frame

- Calibration Parameters

- Outputs to calibrate

- Historical data

- Calibration targets

- Output Variables

- Morbidity by age

- Mortality by age

- Economic effects

- Output Displays

- Plots

- Map Displays 


\section{Computational Framework}

- Differential Equation Models

- Hybrid Models

- Data driven differential equation models

- Agent-Based Models

- Discrete-time stochastic agent models

- Modeling all agents

- Modeling only infected agents

- Event-driven agent models 


\section{Overview of the Ontology}

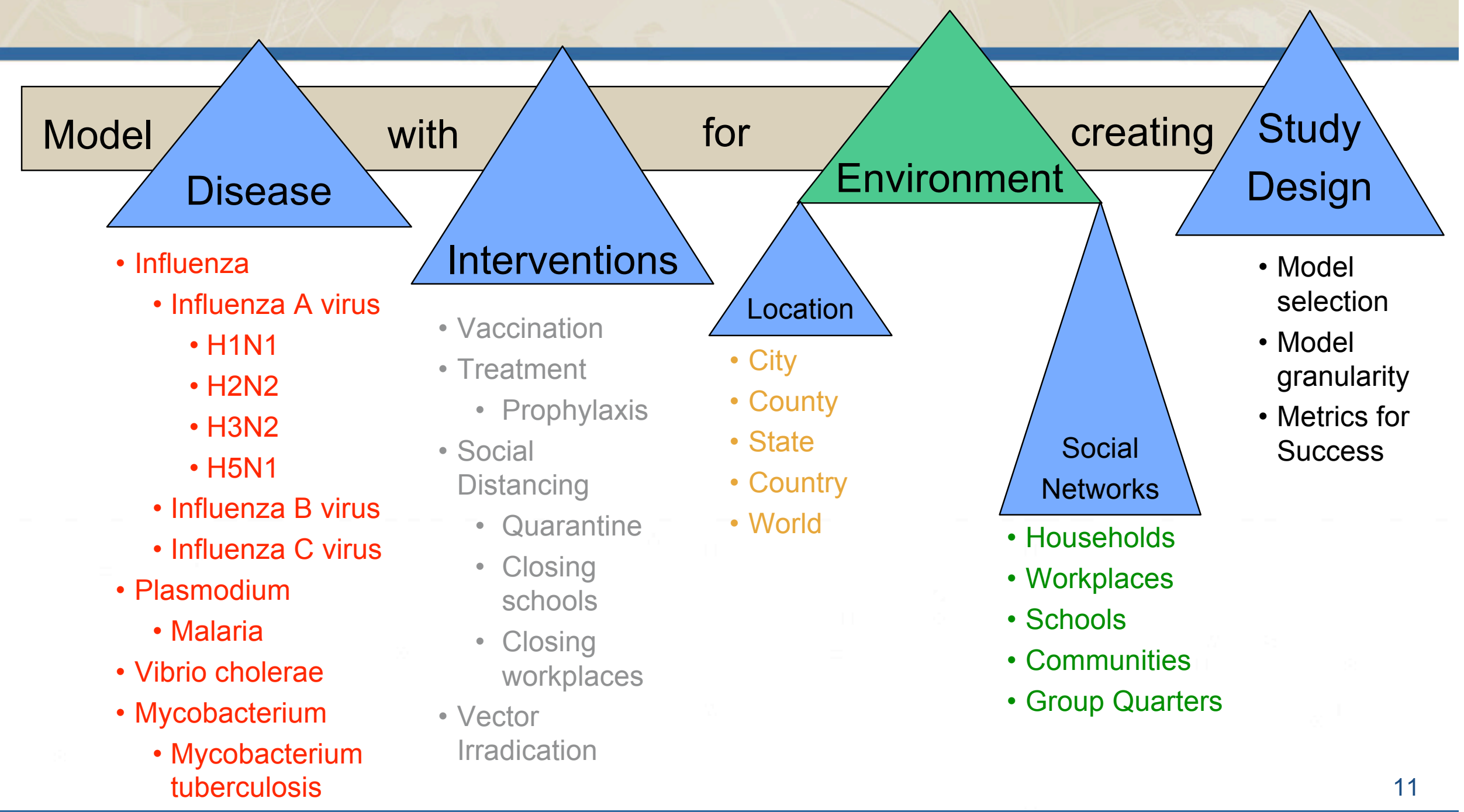




\section{Generic Class Rules}

- Part_Of structures

- Every study and document appears in exactly one sub element

- Each part has a menu

- Top level structures are parts: disease, interventions, environment, study design

- Location and social networks are parts of environment

- (Choice) Is_A structure

- A study or document appears in exactly one sub element

- Exactly one item can be selected from the menu

- Disease and Geographic Location are choices

- (Option) Is_A structure

- A study or document appears in some or none of the sub elements

- Any number of items can be selected from the menu

- Interventions and Social Networks are options 


\section{Class Structures for Taxonomies}

- Model search menu generation

- Study and documentation query generation

- Mapping of data to the taxonomies

- Studies

- Documentation (Historical data for calibration)

- Output visualizations

- Ontology structure validation

- Uniqueness of the mappings

- Completeness of the mappings 


\section{Example Taxonomy Structure}

- <Parts>

- <Ontoobj id="IN:0000001" name="inputs" label="Static Parameters for a Study" >

- <Parts>

+ <Ontoobj id="ID:0000098" name="infectious disease" label="collections of etiologically connected cases of infectious disease in a given population">

+ <OntoObj id="MD:0000001" name="geographic data" label="Geographic data" >

- <Ontoobj id="MD:0000002" name="Social Groups" label="Social Goups">

$-<$ Parts>

- <OntoObj id="DD:0010000" name="households" label="Family Household Groups">

- <Choices>

+ <OntoObj id="DD:0010001" name="No household model" label="No household model" $>$

+ <OntoObj id="DD:0010002" name="Family households" label="Family household">

+ <Ontoobj id="DD:0010003" name="Neighborhood and households" label="Neighborhood and

household groups">

$</$ Choices $>$

$</$ OntoObj>

- <OntoObj id="DD:0020000" name="school groups" label="School Groups">

- <Choices>

- <Ontoobj id="DD:0020001" name="No school groups" label="No school groups">

$<$ Study id="40" co="14" disease="Pandemic Flu" location="World" label="GlobalModel"

method="GlobalModel V1" math="stochastic, equation-based epidemic model"

documentation $="$../Documentation/RTI

Publications/Global_Epidemic_Model_Manual.htm\#LinkTarget_7514" /> 


\section{Taxonomy Dependencies}

- RDF-like relations between taxonomies

- Define menu constraints

- System implements dynamic menu options

- Dependency functions

- Option/Choice T1 requires Option/Choice T2

- Disease Malaria requires Intervention Vector Control Options

- Choice T1 prevents Option/Choice T2

- Intervention Group Quarters Quarantine prevents No Group Quarters Option 


\section{Application: User Interface to the MIDAS Repository}

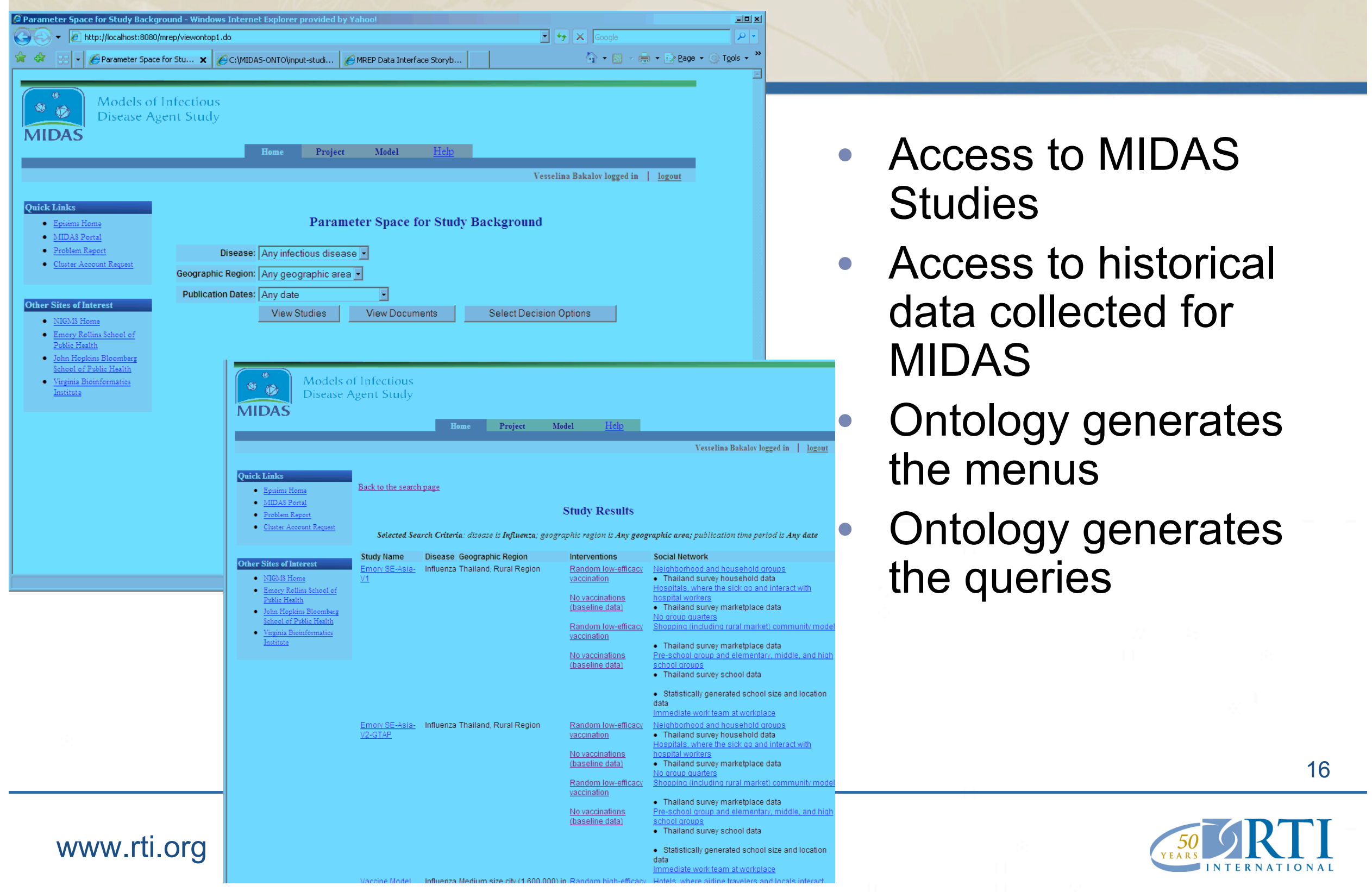




\section{Conclusions}

- Infectious disease models are a good subject for an ontology for epidemiology

- The models and historical data can be tagged by multiple taxonomies

- An ontology provides a good basis for searching a repository of epidemiology models

- An ontology captures the relationships between taxonomies that can reduce the search space

- The ability to link to other ontologies through standard taxonomies is key to leveraging additional data sources 